\section{Dienstärzte als notfallmedizinische Grundversorger} Die Plattform Rettungswesen der FMH ist das Forum der ärztlichen Fachgesellschaften, die an der präklinischen Notfallversorgung beteiligt sind. Deshalb sind auch der Interverband für Rettungswesen IVR und der Koordinierte Sanitätsdienst der Armee vertreten. Das Leitbild der FMH zum Rettungswesen der Schweiz wurde im August 2010 publiziert. Es ist ein wegweisendes Papier, das Leitlinien aufzeigt, um die professionelle Notfallhilfe, auch bei Grossschadens-Ereignissen und Katastrophen, gesamtschweizerisch einheitlich und rechtlich verbindlich zu regeln.

Die FMH setzt sich dafür ein, dass die Notfallversorgung gemeinsam durch Notärzte bzw. speziell ausgebildete Hausärzte einerseits und Rettungssanitäter andererseits gewährleistet ist. Es wird bekanntermassen immer schwieriger, den ärztlichen Nachwuchs für den Notfalldienst zu motivieren; dabei gehört doch die Mitwirkung am Notfalldienst zu den notwendigen Pflichten und zum Kerngeschäft der Grundversorger. Eine Notfallversorgung ohne Integration der niedergelassenen Hausärzte ist heute nicht möglich. Diesem Umstand haben auch die Verantwortlichen des Schweizerischen Instituts für Weiter- und Fortbildung SIWF mit der Integration des Dienstarztkurses ins Weiterbildungsangebot der Grundversorger Rechnung getragen.

Die Plattform Rettungswesen der FMH unterstützt die Grundversorger in ihrer Funktion als Dienstärzte. Um dem drohenden Rückzug der Grundversorger aus der Notfallmedizin entgegenzusteuern, muss die Politik dringend die Hausarztmedizin auch für die Versorgung von Notfallpatienten aufwerten.

Dr. med. Ernst Gähler, Vizepräsident, Verantwortlicher Rettungswesen FMH

\title{
Dienstärzte als notfallmedizinische Grundversorger sind wichtige Partner für das Rettungswesen
}

Bruno Durrera, Wolfgang Ummenhofer ${ }^{\text {, }}$, Heinz Zimmermann

(alphabetisch)

a Dr. med., Facharzt für Allgemeinmedizin FMH, Notarzt SGNOR, Sportmedizin SGSM, Lauterbrunnen

b Prof. Dr. med., Departement Anästhesie, Universitätsspital Basel

c Prof. Dr. med., Direktor Notfallzentrum, Inselspital, Bern
Korrespondenz: Prof. Dr. med. Wolfgang Ummenhofer Universitätsspital Basel Departement Anästhesie CH-4031 Basel

wummenhofer@uhbs.ch
Die Kantone sind gesetzlich verpflichtet, die notfallmedizinische Versorgung überall und jederzeit sicherzustellen. Im Bereich der ärztlichen Grundversorgung haben sie diese Aufgabe an die kantonalen Ärztegesellschaften delegiert.

In Land- und Bergregionen ohne Notarztnetz sollen grundversorgende Ärztinnen und Ärzte mit der Zusatzausbildung «Dienstarzt» für vitalbedrohliche Notfälle eingesetzt werden, um die Zeit bis zum Eintreffen der Boden- oder Luftrettung zu überbrücken. Die SGNOR (Schweizerische Gesellschaft für Notfall- und Rettungsmedizin) unter Supervision der FMH bietet Dienstarztkurse für Grundversorger an (www.fmh.ch $\rightarrow$ SERVICES $\rightarrow$ Plattform Rettungswesen $\rightarrow$ Dienstarzt - Ärztlicher Notfalldienst). Die Ausbildung der Not- und Dienstärzte unterscheidet sich vor allem durch die Invasivität der instruierten präklinischen Massnahmen. Aber auch Agglomerationen profitieren von einer Vernetzung der Dienstärzte mit den Notarztdiensten, weil sich auch initial «harmlos» klingende Hausbesuche zu lebensbedrohlichen Notfallsituationen entwickeln können. Für eine optimale Triagierung der Notfallpatienten müssen die Sanitätsnotrufzentralen und die Ärzte-Notrufsysteme eng zusammenarbeiten.

Nicht vitalbedrohliche, ambulant versorgbare medizinische Notfälle ausserhalb der Sprechstundenzeiten können durch Ärztenotrufzentralen triagiert und an die nächste diensttuende Grundversorgerstelle überwiesen werden. Dafür haben sich neue eigenständige Hausarzt-Notfallstationen zum Teil in
Zusammenarbeit mit Notfallabteilungen von Spitälern etabliert.

Bei potentieller Vitalgefährdung muss die Alarmierung aber über Telefon 144 gehen. Die Sanitätsnotrufzentrale kann dann die initialen Glieder der «Rettungskette» wie Laien- und Ersthelfer optimal mit Dienstärzten und der professionellen Bodenoder Luftrettung koordinieren.

Die Notfallversorgung der Bevölkerung - eine Kernkompetenz der Hausarztmedizin - ist aber aufgrund des Hausärztemangels zunehmend gefährdet. So belastet die starke Abnahme der Hausärzte den Notfalldienst in der Peripherie zunehmend und

Die Plattform Rettungswesen der FMH entwickelt als Forum der ärztlichen Fachgesellschaften Leitlinien für eine einheitliche und verbindliche präklinische Notfallversorgung, auch bei Grossereignissen. Die FMH setzt sich dafür ein, dass die Notfallversorgung gemeinsam durch Notärzte bzw. speziell ausgebildete Hausärzte einerseits und Rettungssanitäter andererseits gewährleistet wird. Im Sommer $\mathbf{2 0 1 0}$ hat die Plattform Rettungswesen ihr Thesenpapier zum Rettungswesen in der Schweiz aus dem Jahr 1996 aktualisiert. (Schweizerische Ärztezeitung Nr. 33/2010, S. $1215 \mathrm{f}$. und www.fmh.ch $\rightarrow$ SERVICES $\rightarrow$ Plattform Rettungswesen). Periodisch soll nun in der Ärztezeitung auf die einzelnen der acht Punkte des neuen Leitbilds näher eingegangen werden. 
macht den Beruf wenig attraktiv, speziell für Frauen mit Familie. Junge Ärztinnen und Ärzte sind heute nicht mehr bereit, eine 24-Stunden-Hausarzt-Dienstbereitschaft kostenlos aufrechtzuerhalten, wenn gleichzeitig die finanzielle Leistungsabgeltung reduziert wird. Das Dienstarztkonzept kann aber nur dort funktionieren, wo es auch Hausärzte gibt. Es droht somit eine subakute notfallmedizinische Unterversorgung der peripheren Gebiete, die mittel- und langfristig auch nicht mehr mit ausländischen Ärztinnen und Ärzten aufgefangen werden kann.

Dieser Entwicklung muss die Politik entgegensteuern: Es braucht zunächst eine klare Absichtserklärung der politischen Entscheidungsträger, die Grundversorger $\mathrm{zu}$ stärken und sie weiterhin vor allem in der Peripherie in die Notfallversorgung einzubinden. Zudem müssen die Kantone die Grundversorger als Dienstärzte für den Dienstarztkurs, für die Pikettleistung und für die Notfallausrüstung entschädigen. Auf dem Land und in den Bergen stellen Gemeinschaftspraxen, Ärztehäuser und Gesundheitszentren zukünftig die Grund- und Notfallversorgung in grösseren Einzugsgebieten sicher. Unbesetzte periphere Arztpraxen können als Gesundheitsposten von Gemeinde- und Spitexschwestern oder Medizinischen Praxisassistentinnen und -assistenten betreut werden. Sie sind mit der nächsten ärztlichen Grundversorgerstelle telemetrisch vernetzt, die auch die Verantwortung für die Aussenstelle trägt.

Die Plattform Rettungswesen der FMH unterstützt die Grundversorger in ihrer Funktion als Dienstärzte. Um dem drohenden Rückzug der Grundversorger aus der Notfallmedizin entgegenzusteuern, muss die Politik dringend die Hausarztmedizin auch für die Versor- gung von Notfallpatienten aufwerten. Hierfür sind im Medizinstudium verstärkt Wissen und Fertigkeiten notfallmedizinscher Kompetenzen zu vermitteln. Die FMH setzt sich dafür ein, dass die Lerninhalte des Dienstarztkurses (DAK) Eingang in die Curricula der Schweizerischen Medizinischen Fakultäten finden.

\section{Zusammenfassung:}

1. Es braucht eine klare Absichtserklärung der Politik, die Grundversorger auch in ihrer notfallmedizinischen Kompetenz zu stärken und sie weiterhin in die Notfallversorgung einzubinden.

2. Überall, in der städtischen Praxis wie auch in ländlichen Regionen, können Grundversorger die Zeit bis zum Eintreffen der Bodenoder Luftrettung überbrücken.

3. In der Peripherie können die Kantone grundversorgende Ärzte mit der Zusatzausbildung «Dienstarzt» für vitalbedrohliche Notfälle einsetzen. Die Plattform Rettungswesen der FMH unterstützt ein solches Dienstarztsystem mit entsprechender Entgeltung und fordert deshalb von der Politik eine Aufwertung und Anerkennung der Hausarztmedizin.

4. Zu diesem Zweck müssen notfallmedizinische Grundkompetenzen schon während des Medizinstudiums erworben werden. Die FMH setzt sich dafür ein, dass die Lerninhalte des Dienstarztkurses (DAK) Eingang in die Curricula der Schweizerischen Medizinischen Fakultäten finden. 